\title{
Colangitis esclerosante secundaria en pacientes críticamente enfermos
}

\author{
Secondary sclerosing cholangitis in critically ill patients
}

Carlos Alberto Peña-Pérez ${ }^{*}$ y Juan Alberto Díaz Ponce-Medrano²

'Unidad de Terapia Intensiva de Adultos; '2Dirección Médica. Hospital General Naval de Alta Especialidad, Secretaría de Marina/Armada de México, Ciudad de México, México

\begin{abstract}
Resumen
La colangitis esclerosante primaria (CEP) es una condición idiopática rara con mecanismos inmunopatogénicos en la que existe una destrucción crónica y progresiva del árbol biliar. La colangitis esclerosante secundaria (CES) es clínicamente comparable a la CEP, pero está causada por procesos específicos que dañan directamente el árbol biliar; ejemplo de esto son enfermedades como la pancreatitis recurrente, las neoplasias de la vía biliar y las anomalías congénitas de la vía biliar, entre otras. Una nueva causa de CES ha sido descrita durante o después de una enfermedad crítica, en muchas ocasiones relacionada con las intervenciones de soporte o con condiciones de ingreso propias de la unidad de terapia intensiva, como son la ventilación mecánica invasiva, los vasopresores, el estado de choque y la sepsis. Esta condición progresa rápidamente a cirrosis, y con frecuencia requiere un trasplante de hígado para su manejo definitivo.
\end{abstract}

PALABRAS CLAVE: Colangitis esclerosante secundaria. Paciente crítico. Colangiopancreatografía retrógrada endoscópica.

\begin{abstract}
Primary sclerosing cholangitis (PSC) is a rare idiopathic condition with immunopathogenic mechanisms where there is chronic progressive destruction of the biliary tree. Secondary sclerosing cholangitis (SSC) is clinically comparable to PSC, but is caused by specific processes which directly damage the biliary tree; examples include recurrent pancreatitis, bile duct malignancy, congenital bile duct abnormalities. A new cause of SSC has been described during or following significant critical illness associated with severe respiratory insufficiency, vasopressor requirement, shock and sepsis. This condition rapidly progresses to cirrhosis, frequently requiring liver transplantation for definitive management.
\end{abstract}

KEY WORDS: Secondary sclerosing cholangitis. Critically ill patient. Endoscopic retrograde cholangiopancreatography.

\author{
Correspondencia: \\ *Carlos Alberto Peña-Pérez \\ Calzada de la Virgen, $s / n$ \\ Col. Presidentes Ejidales 1ra Sección, \\ Del. Coyoacán \\ C.P. 04470, Ciudad de México, México \\ E-mail: galeno_78@yahoo.com.mx
}

Fecha de recepción: 18-08-2017

Fecha de aceptación: 14-02-2018

DOI://dx.doi.org/10.24875/CIRU.M18000003
Cir Cir. 2018;86:56-62

Disponible en PubMed www.cirugiaycirujanos.com 


\section{Introducción}

El progreso médico y tecnológico en la medicina de cuidados críticos ha permitido aumentar las tasas de sobrevida de los pacientes con lesiones que amenazan la vida. Sin embargo, este incremento en la tasa de sobrevida de los enfermos graves se acompaña del surgimiento de nuevas enfermedades «adquiridas o relacionadas" con los cuidados intensivos. En los últimos años se han registrado cada vez más informes de nuevas enfermedades relacionadas con los cuidados intensivos.

El desarrollo de colangitis esclerosante secundaria (CES) en pacientes críticamente enfermos (CES-PCE) se observó por primera vez en pacientes con quemaduras graves y politraumatismo, pero posteriormente también se ha descrito en otros eventos críticos agudos que ponen en peligro la vida.

La CES-PCE se caracteriza por colestasis y necrosis de los conductos biliares, y puede ser diagnóstica a partir de hallazgos colangiográficos típicos. Esta enfermedad parece ser irreversible y de progresión rápida a cirrosis biliar e insuficiencia hepática. Sin excepción, el historial médico de todos los casos de CES-PCE revela el tratamiento previo en la unidad de terapia intensiva (UTI) ${ }^{1-4}$. No hay duda de que los factores desencadenantes de la CES-PCE se encuentran en el intervalo de tiempo entre el evento inicial que amenaza la vida y la ocurrencia de la colestasis. Si esta afección es causada por la propia lesión inicial o por el tratamiento brindado en la UTI, no está aún claro. Las opciones terapéuticas para la CES-PCE son limitadas, la tasa de mortalidad durante la estancia inicial en la UTI es alta y muchos pacientes que sobreviven tienen que someterse a trasplante hepático.

Así como la polineuropatía del enfermo grave, la CES-PCE es una complicación de la enfermedad crítica grave y de su manejo exitoso. La CES-PCE es una causa importante de insuficiencia hepática aguda inexplicable en los pacientes de la UTI, y contribuye a pobres desenlaces. En la CES-PCE, ya sea la propia enfermedad crítica o su manejo pueden convertir los conductos biliares previamente sanos en conductos gravemente destruidos al cabo de pocos días. Este daño en los conductos biliares se inicia dentro de los primeros 7 días tras el ingreso a la UTI. Varios factores de riesgo se han considerado, siendo los más relevantes el estado de choque, la hipotensión arterial, las dosis altas de fármacos vasopresores, la sepsis, la nutrición parenteral total y las estrategias de ventilación mecánica invasiva con presión positiva al final de la espiración (PEEP) $>10 \mathrm{cmH}_{2} \mathrm{O}$. Sin embargo, permanece el debate respecto a cuál de estos factores de riesgo es el principal promotor de daño en los conductos biliares en la CES-PCE.

Siguiendo los criterios de Hill, los posibles factores de riesgo pueden ser:

- Presentes antes del inicio de la colestasis (temporalidad).

- Comunes a todos los pacientes con este problema (consistencia).

- Biológicamente plausibles; por ejemplo, capaces de conducir la patogénesis de la «bilis tóxica» o "colangiopatía isquémica" (plausible).

Las pruebas de temporalidad, consistencia y plausibilidad son una condición sine qua non para la investigación de los mecanismos causales ${ }^{5}$.

La destrucción de los conductos biliares en la CESPCE parece ser la consecuencia de un solo evento, durante el cual un gran número de colangiocitos son dañados de manera directa e irreversible. El daño directo a los colangiocitos puede ser inducido por isquemia/hipoxia o por las propiedades detergentes de la llamada «bilis tóxica». La isquemia y la bilis tóxica son concebibles como dos mecanismos fisiopatológicos subyacentes de la CES-PCE.

\section{Concepto de la colangiopatía isquémica}

En comparación con los hepatocitos, que tienen un doble suministro de sangre oxigenada, el epitelio biliar se irriga solo a través de las arterias hepáticas. En consecuencia, el epitelio biliar es más susceptible a la isquemia que los hepatocitos. Antes de la década de 1960, la colangiopatía isquémica era virtualmente desconocida y nuestra información clínica sobre las lesiones inducidas a los conductos biliares por isquemia se basaba casi exclusivamente en la experiencia con el trasplante hepático ${ }^{6}$ (Fig. 1).

Poco después del trasplante hepático, la interrupción del suministro de sangre arterial (p. ej., por trombosis de la arteria hepática) resulta en la necrosis de los conductos biliares. Un mayor soporte al concepto de colangiopatía isquémica vino de observaciones realizadas en los años 1980, demostrando que la administración de floxuridina en la arteria hepática resulta en necrosis de los conductos biliares y colangitis esclerosante ${ }^{7,8}$. La necrosis isquémica del epitelio biliar puede ocurrir cuando el suministro de sangre a los conductos biliares se altera, ya sea la macrocirculación o la microcirculación. 


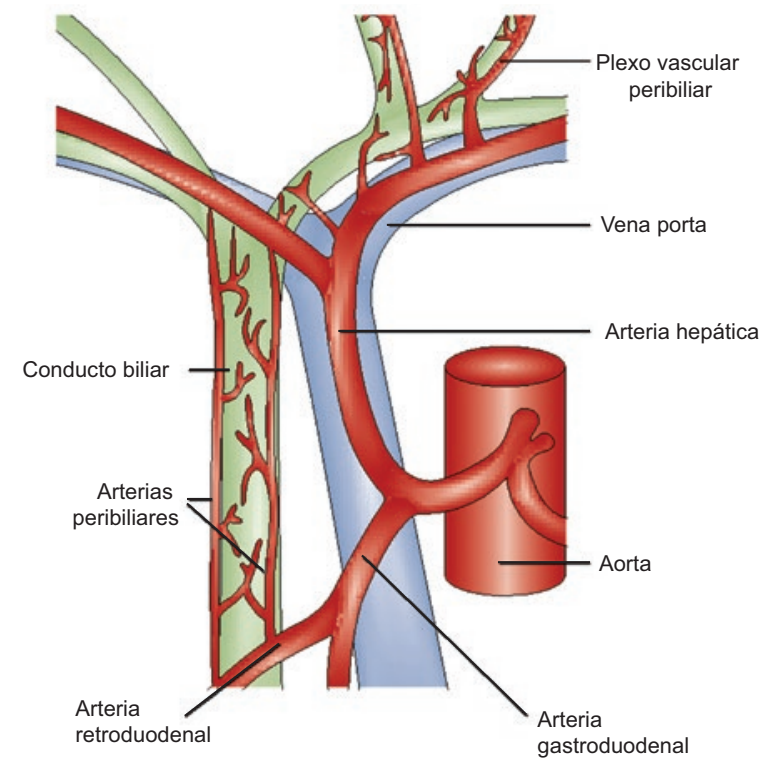

Figura 1. Características de la irrigación de los conductos hepáticos.

\section{Concepto de la bilis tóxica}

En ausencia de mecanismos protectores, las propiedades detergentes de los ácidos biliares hidrófobos destruirán inmediatamente la membrana celular lipídica de los colangiocitos. Un mecanismo protector importante es la secreción hepatocelular de fosfolípidos, que forman micelas mixtas protectoras junto con los ácidos biliares. Además, la secreción biliar de bicarbonato da como resultado la formación de una película alcalina protectora sobre la membrana apical de los colangiocitos, contribuyendo este mecanismo como parte de la estrategia de defensa ${ }^{9}$. Un equilibrio finamente ajustado entre los ácidos biliares y los mecanismos protectores es imprescindible para mantener la integridad del epitelio de los conductos biliares. Por lo tanto, las alteraciones en este equilibrio darán como resultado un grave daño al epitelio biliar y el desarrollo de colangitis esclerosante ${ }^{10-13}$. Tales alteraciones pueden ser el resultado de trastornos funcionales del sistema de transporte hepatobiliar.

\section{Alteraciones macrocirculatorias como promotoras de la colangiopatía isquémica}

En el caso de un colapso circulatorio incipiente con hipotensión arterial es de esperar un deterioro de la perfusión hepática con alteraciones posteriores del suministro sanguíneo a los conductos hepáticos, dado que la perfusión hepática es capaz de mantenerse por autorregulación solo por un periodo limita$\mathrm{do}^{14}$. El estado de choque con disminución de la presión arterial es un fenómeno común en los pacientes ingresados a las UTI, con una frecuencia de hasta el $58 \%$ según reportes recientes ${ }^{15}$.

Cuando la administración de soluciones parenterales no logra restablecer la presión arterial sistémica de manera adecuada, y ante una situación clínica crítica, está indicado iniciar el manejo con agentes vasoactivos. Sin embargo, dado que el aumento de la presión arterial sistémica no siempre se correlaciona con una mejor perfusión hepatoesplácnica, la isquemia hepática prolongada puede quedar enmascarada. Los agentes vasoactivos de uso común, como la epinefrina, la norepinefrina, la dopamina y la dobutamina, tienen la capacidad de incrementar la presión arterial sistémica, pero cada uno con diferentes efectos sobre el flujo sanguíneo hepatoesplácnico. La dopamina no solo aumenta el flujo sanguíneo mesentérico, sino que aparentemente también tiene un efecto positivo en la perfusión hepática ${ }^{16}$. En contraste, la epinefrina y la norepinefrina tienen un efecto negativo dependiente de la dosis sobre la perfusión de los órganos viscerales. Debido a sus propiedades vasoconstrictoras, la norepinefrina tiene el potencial de disminuir el flujo sanguíneo esplácnico. La epinefrina, en particular, es conocida por su efecto deletéreo sobre la perfusión esplácnica en pacientes con sepsis ${ }^{17,18}$.

\section{Alteraciones microcirculatorias como promotoras de la colangiopatía isquémica}

Como se sabe a partir de modelos experimentales, el daño isquémico del conducto biliar aumenta a medida que disminuye el tamaño de las arterias ocluidas $^{19-21}$. Esto indica que las alteraciones microcirculatorias que afectan al plexo peribiliar tienen un significado clínico particular. La pregunta de por qué la CES-PCE ocurre más a menudo en pacientes quirúrgicos todavía no se ha respondido. Dos tercios de los casos publicados de CES-PCE corresponden a pacientes con traumatismos, quemados y sometidos a cirugía mayor. El politraumatismo y la cirugía mayor suelen acompañarse de un daño extenso en los tejidos. Los estudios clínicos han demostrado que este daño tisular puede ser un iniciador del estado de hipercoagulación ${ }^{22-27}$. Esto podría contribuir a la oclusión del plexo peribiliar. El daño isquémico del conducto biliar se ha encontrado frecuentemente en una serie de condiciones conocidas por estar asociadas a un estado de hipercoagulación, como el síndrome antifosfolípido, la hemoglobinuria paroxística nocturna y el lupus eritematoso sistémico, entre otras ${ }^{28-32}$. 
Otros factores importantes que afectan a la microcirculación son el incremento de la viscosidad sanguínea y la agregación de los glóbulos rojos. Los traumatismos, las quemaduras y la cirugía mayor son condiciones que pueden aumentar la viscosidad plasmática. Además, la destrucción extensa del músculo, los accidentes con energía eléctrica y el síndrome de isquemia-reperfusión se asocian a menudo con una liberación marcada de mioglobina, lo que puede condicionar un aumento de la viscosidad plasmática ${ }^{33}$. La infusión parenteral de soluciones coloidales, como el hidroxietil almidón, también puede aumentar la viscosidad sanguínea. Los efectos desfavorables de estas soluciones sobre el flujo sanguíneo del plexo peribiliar han sido reportados en estudios clínicos y apoyados por otros de carácter experimental ${ }^{34,35}$.

En los últimos años se ha puesto de manifiesto la existencia de riesgos secundarios a la transfusión de glóbulos rojos, principalmente por los efectos de almacenamiento de estos. Los glóbulos rojos almacenados se adhieren al endotelio o pierden su capacidad de deformabilidad, resultando en una obstrucción de la microcirculación ${ }^{36-38}$. Estas observaciones sugieren que, aparte de la hipotensión grave, ciertos trastornos microcirculatorios adicionales pueden estar involucrados en la patogénesis de la CES-PCE. Posiblemente, la hipotensión arterial grave solo puede tener implicaciones sobre la vía biliar cuando ocurre en combinación con trastornos microcirculatorios adicionales.

\section{La ventilación mecánica invasiva como conductora de la colangiopatía isquémica}

Estudios experimentales indican que la ventilación mecánica con PEEP elevada para fines de protección alveolar prolongada tiene efectos negativos sobre la microcirculación en el tracto gastrointestinal. Sobre la base de estos estudios, se sospecha que, en particular, una PEEP elevada contribuye a la isquemia biliar, y que los pacientes con un bajo índice de oxigenación corren el riesgo de desarrollar CES-PCE ${ }^{2,39}$.

\section{Síndrome de respuesta inflamatoria sistémica y sepsis como conductores de «bilis tóxica» para el desarrollo de colangitis esclerosante secundaria en pacientes críticamente enfermos}

Las citocinas proinflamatorias, como el factor de necrosis tumoral alfa y las interleucinas 1 y 6 , regulan negativamente la función o la expresión de los transportadores hepatobiliares. Modelos animales indican, por ejemplo, que la bomba canicular de bilirrubina conjugada es sensible a las citocinas, y que el mRNA de la bomba de exportación de sales biliares es regulada de manera negativa por la influencia de mediadores inflamatorios ${ }^{40-42}$. Estos datos han sido confirmados en hepatocitos humanos. Sin embargo, los datos sobre la bomba de exportación de fosfolípidos no son concluyentes, pero algunos estudios en modelos de inflamación experimental sugieren una disminución de su expresión hepática ${ }^{43}$.

En cuanto al transportador biliar, las citocinas proinflamatorias inhiben la actividad del intercambiador aniónico $A E 2^{44}$. En el entorno clínico, el síndrome de respuesta inflamatoria y la sepsis reflejan un ambiente rico en la liberación de citocinas proinflamatorias ${ }^{45}$.

\section{Síntomas clínicos y diagnóstico}

En la etapa inicial de la CES-PCI, los síntomas clínicos son raros e inespecíficos y el único indicador de colestasis son los resultados anormales en las pruebas de funcionamiento hepático. En particular, las concentraciones séricas de fosfatasa alcalina (FA) y de gamma-glutamil transferasa (GGT) son muy elevadas; sin embargo, ningún parámetro específico indica la presencia de CES-PCE temprana, y por lo tanto el diagnóstico suele ser tardío. A medida que la enfermedad avanza, la presencia de colestasis persistente puede ser indicativa de CES-PCE. Los síntomas clínicos más comunes en esta etapa avanzada son ictericia, prurito y malestar abdominal localizado en el cuadrante superior derecho del abdomen. La infección biliar ascendente está presente en casi todos los pacientes con CES, y típicamente se observan episodios recurrentes de colangitis bacteriana ${ }^{46-48}$.

En la serie de 16 pacientes publicada por Leonhardt, et al. ${ }^{49}$, el inicio de la colestasis se observó 7 días (media) tras el inicio del evento clínico que amenazaba la vida. La GGT fue el primer parámetro que se elevó, y la FA le siguió pocos días después. La hiperbilirrubinemia pudo observarse después de una media de $15.5 \pm 10$ días (mediana 13 días) y fue, por lo tanto, el último signo de la enfermedad detectado. Esta secuencia característica de GGT, FA y elevación de la bilirrubina se observó en 13 de los 16 pacientes. La colestasis alcanzó un pico máximo después de 31 \pm 14 días. Los valores de la GGT alcanzaron un máximo de unas 20-50 veces el límite superior de la normalidad, mientras que la elevación de la FA fue 
menos pronunciada (5-21 veces el límite superior de la normalidad). Los valores de alanina aminotransferasa y de aspartato aminotransferasa fueron moderadamente elevados. La colestasis inicial se acompañó de una elevación transitoria de la creatinina sérica en la mitad de los pacientes ${ }^{49}$.

Después de obtener resultados anormales en las pruebas de funcionamiento hepático, la ultrasonografía (USG) se utiliza para identificar a pacientes con sospecha de enfermedad de la vía biliar o de anormalidades estructurales hepáticas. La USG tiene la capacidad de detectar cálculos biliares, dilatación del conducto biliar o la presencia de un absceso hepático en los pacientes en que la obstrucción biliar crónica es la principal causa de la colangitis esclerosante. Sin embargo, en los pacientes con CES sin obstrucción ni dilatación de la vía biliar, la USG no facilita el diagnóstico.

La colangiopancreatografía retrógrada endoscópica (CPRE) es el método de referencia para el diagnóstico en todos los pacientes con sospecha de CES. Los hallazgos típicos de la CPRE en la CES son lesiones en los conductos biliares con estenosis multifocales y segmentos intermedios de conductos normales o dilatados que confieren a los conductos biliares intrahepáticos un aspecto de "cuentas». Los pequeños conductos biliares periféricos pueden mostrar un pobre llenado u una obliteración completa, dando a la vía biliar intrahepática una apariencia de «árbol podado".

La CPRE presenta tres patrones colangiográficos dependiendo del estadio en que se encuentre la enfermedad (Fig. 2):

- Fase temprana: formación de cilindros biliares. La etapa temprana de la enfermedad se caracteriza por la presencia de múltiples defectos de llenado intraductales, semejantes a una "cinta» del tracto biliar.

- Fase intermedia: destrucción progresiva de la vía biliar intrahepática. El curso posterior de la enfermedad se caracteriza por una rápida y progresiva destrucción de los conductos biliares intrahepáticos, más allá de la segunda bifurcación. La destrucción de los conductos biliares intrahepáticos por necrosis epitelial se observa en la CPRE como conductos de contornos irregulares y múltiples defectos de llenado en los bordes de la pared.

- Fase tardía: obliteración de los conductos biliares intrahepáticos (imagen del «árbol podado»). Como resultado de la destrucción progresiva, las ramas de los conductos biliares intrahepáticos son eventualmente «borradas», dejando solo un sistema biliar central rudimentario. En la CPRE, el realce con contraste de los conductos biliares solo se observa por debajo de la segunda bifurcación. En esta última etapa, la imagen colangiográfica es similar a la de un «árbol podado». Al menos un lado del sistema biliar está obliterado, pero en su mayoría ambos lados se ven afectados ${ }^{49}$.

La colangiopancreatografía por resonancia magnética es menos invasiva que la CPRE, pero no es tan útil para la detección de leves lesiones tempranas ${ }^{50}$.

\section{Complicaciones de la colangitis esclerosante secundaria en pacientes críticamente enfermos}

La CES-PCE conlleva un alto riesgo de complicaciones hepatobiliares, destacando entre ellas la colecistitis acalculosa aguda, la perforación de la vesícula biliar, el absceso hepático y la colangitis séptica ${ }^{49}$.

A tal respecto, Voigtländer, et al. ${ }^{51}$, en el año 2010, evaluaron en un estudio prospectivo a 169 pacientes (72 con diagnóstico de CES-PCE y 97 con diagnóstico de colangitis esclerosante primaria [CEP]). En su estudio obtuvieron muestras de bilis mediante CPRE de cada paciente para realizar cultivos. De las muestras de bilis procesadas, resultaron con cultivos positivos el $81.1 \%$ de los casos. El $94.4 \%$ de las muestras (68/72) fueron positivas en los pacientes con CESPCE, en comparación con el $71.1 \%$ (69/97) de los pacientes con CEP. El crecimiento monomicrobiano fue más frecuente en los pacientes con CES-PCE. Las cepas grampositivas se aislaron más que las cepas gramnegativas en ambas cohortes. El microorganismo más abundantemente aislado entre los grampositivos fue Enterococcus spp. (36\%), y entre los gramnegativos fueron Escherichia coli (13\%), Klebsiella spp. (9\%) y Pseudomonas spp. (6\%). Candida albicans se aisló en el $11 \%$ de los casos. De acuerdo con estos resultados, una terapia antimicrobiana inicial con vancomicina y piperacilina-tazobactam \pm fluconazol puede ser eficaz en la mayoría de los pacientes con CES-PCE ${ }^{51}$.

\section{Progresión y necesidad de trasplante hepático}

Dos aspectos contribuyen a la corta latencia entre el inicio de la colestasis y la necesidad de trasplante en los casos reportados de CES-PCE. En primer lugar, el curso clínico e histológico de la fibrosis hepática es 


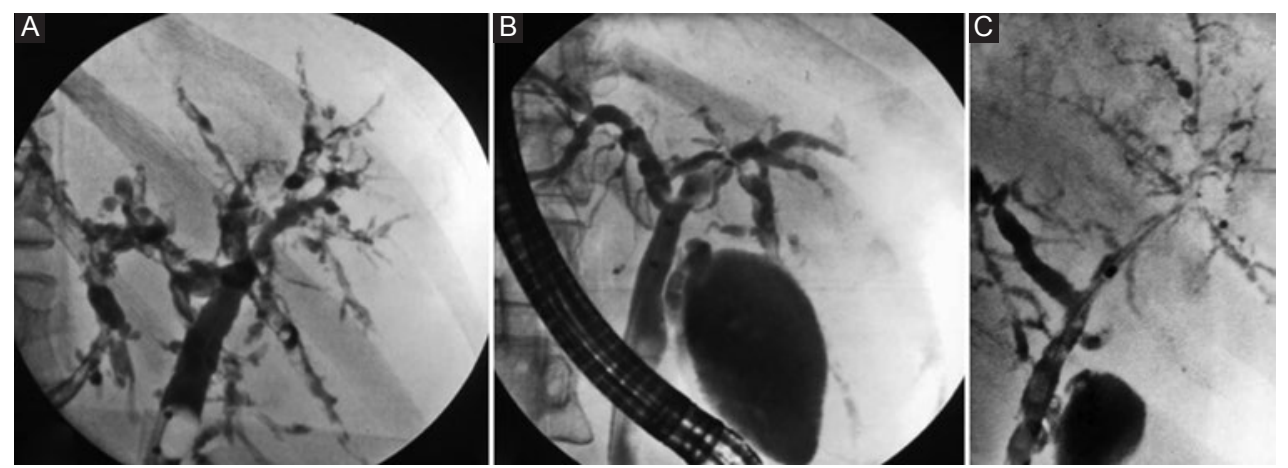

Figura 2. Patrones colangiográficos. A: fase temprana. Defectos de llenado por presencia de cilindros biliares. B: fase intermedia. Defectos de llenado por la destrucción de los conductos biliares, generando imágenes similares a "cuentas». C: fase tardía. Obliteración de los conductos biliares intrahepáticos con defecto de llenado que semeja a un "árbol podado».

de una progresión inusualmente rápida, en pocos meses. Esta rápida y extensa respuesta fibrótica puede ser explicada por la gran destrucción inicial de los colangiocitos de los conductos biliares intrahepáticos. En segundo lugar, debido al riesgo de colangitis séptica y la destrucción irreversible de los conductos biliares por un cuadro de colangitis recurrente, es necesario considerar como prioridad urgente el trasplante para estos pacientes ${ }^{52-54}$. La sobrevida posterior al trasplante hepático es variable; se ha reportado una sobrevida al año del $85 \%$, y a los 3 años del $83 \%{ }^{49}$.

\section{Conclusiones}

La CES-PCE puede ser causada por un amplio espectro de eventos que conducen a una lesión grave de la vía biliar intrahepática y extrahepática. Es una enfermedad progresiva que, en la mayoría de los casos, implica la destrucción del árbol biliar y da como resultado una reducción significativa de la esperanza de vida. Si bien la patogénesis de la CES está típicamente asociada con una causa conocida, como obstrucción crónica, lesiones isquémicas del conducto biliar o daño secundario a infecciones o toxinas, la variante relacionada con el enfermo en estado crítico, que no está asociada con procesos patológicos subyacentes ni con obstrucciones de la vía biliar, es una nueva enfermedad cada vez más reconocida. Los pacientes con CESPCE tienen una velocidad aún más rápida de progresión de la enfermedad que los pacientes de las formas "Clásicas» de CES. Durante las etapas iniciales de la enfermedad, las características clínicas son inespecíficas y no permiten diferenciarla de manera confiable de la ictericia inducida por endotoxinas, que es muy frecuente en los pacientes graves. El diagnóstico precoz es, por lo tanto, particularmente difícil. La CPRE es el único método confiable para diagnosticar la CES-PCE, pero este procedimiento invasivo por lo general es evitado en los pacientes críticamente enfermos. Así, la CES-PCE probablemente sea subdiagnosticada en muchos casos, y la verdadera prevalencia de esta enfermedad puede estar sustancialmente infraestimada. Una mayor comprensión de la patogénesis de la CESPCE abre el camino a la prevención y a nuevas estrategias de tratamiento de esta catastrófica afección.

\section{Responsabilidades éticas}

Protección de personas y animales. Los autores declaran que para esta investigación no se han realizado experimentos en seres humanos ni en animales.

Confidencialidad de los datos. Los autores declaran que en este artículo no aparecen datos de pacientes.

Derecho a la privacidad y consentimiento informado. Los autores declaran que en este artículo no aparecen datos de pacientes.

\section{Financiamiento}

Para el desarrollo del presente trabajo no se contó con financiamiento alguno para los autores.

\section{Conflicto de intereses}

Los autores nos declaramos sin conflicto de intereses para el desarrollo y publicación del presente trabajo.

\section{Bibliografía}

1. Schmitt M, Kölbel CB, Müller MK, Verbeke CS, Singer MV. Sclerosing cholangitis after burn injury. Z Gastroenterol. 1997;35:929-34

2. Gelbmann $\mathrm{CM}$, Rümmele $\mathrm{P}$, Wimmer $M$, Hofstädter $F$, Gohlmann B, Endlicher $\mathrm{E}$, et al. Ischemic-like cholangiopathy with secondary sclerosing cholangitis in critically ill patients. Am J Gastroenterol. 2007;102:1221-9. 
3. Engler S, Elsing C, Flechtenmacher C, Theilmann L, Stremmel W Stiehl A. Progressive sclerosing cholangitis after septic shock: a new variant of vanishing bile duct disorders. Gut. 2003;52:688-93

4. Jaeger C, Mayer G, Henrich R, Gossner L, Rabenstein T, May A, et al. Secondary sclerosing cholangitis after long-term treatment in an intensive care unit: a clinical presentation, endoscopic findings, treatment, and follow-up. Endoscopy. 2006;38:730-4.

5. Hill $A B$. The environment and disease. Association or causation? Proc R Soc Med. 1965;58:265-300.

6. Sherlock S. Chronic cholangitides: aetiology, diagnosis, and treatment BMJ. 1968;3:515-21.

7. Hohn D, Melnick J, Stagg R, Altman D, Friedman M, Ignoffo R, et al Biliary sclerosis in patients receiving hepatic arterial infusion of floxuridine. J Clin Oncol. 1985;3:98-102.

8. Ludwig J, Kim CH, Wiesner RH, Krom RA. Floxuridine-induced sclerosing cholangitis: an ischemic cholangiopathy? Hepatology. 1989;9:215-8.

9. Beuers U, Hohenester S, de Buy Wenniger LJ, Kremer AE, Jansen PL Elferink RP. The biliary $\mathrm{HCO}(3)(-)$ umbrella: a unifying hypothesis on pathogenetic and therapeutic aspects of fibrosing cholangiopathies. $\mathrm{He}$ patology. 2010;52:1489-96.

10. Smit JJ, Schinkel AH, Oude Elferink RP, Groen AK, Wagenaar E, van Deemter L, et al. Homozygous disruption of the murine mdr2 P-glycoprotein gene leads to a complete absence of phospholipid from bile and to liver disease. Cell. 1993;75:451-62.

11. Mauad TH, van Nieuwkerk CM, Dingemans KP, Smit JJ, Schinkel AH, Notenboom RG, et al. Mice with homozygous disruption of the mdr2 P-glycoprotein gene. A novel animal model for studies of nonsuppurative inflammation cholangitis and hepato-carcinogenesis. Am $\mathrm{J}$ Pathol. 1994:145:1237-45.

12. Popov Y, Patsenker E, Pickert P, Trauner M, Schuppan D. Mdr2 (Abcb4)-/- mice spontaneously develop severe biliary fibrosis via massive dysregulation of pro- and antifibrogenic genes. J Hepatol. 2005; 43:1045-54.

13. Strazzabosco M, Spirli C, Okolicsanyi L. Pathophysiology of the intrahepatic biliary epithelium. J Gastroenterol Hepatol. 2000;15:244-53.

14. Lautt WW. Regulatory processes interacting to maintain hepatic blood flow constancy: vascular compliance, hepatic arterial buffer response, hepatorenal reflex, liver regeneration, escape from vasoconstriction. $\mathrm{He}-$ patol Res. 2007;37:891-903.

15. Bahloul M, Samet M, Chaari A, Ben Aljia N, Ben Mbarek MN, Chelly H, et al. Use of catecholamines for shock a continuous debate! Tunis Med. 2012;90:291-9.

16. Hiltebrand LB, Krejci V, Sigurdsson GH. Effects of dopamine, dobutamine, and dopexamine on microcirculatory blood flow in the gastrointestina tract during sepsis and anesthesia. Anesthesiology. 2004;100:1188-97.

17. Meier-Hellmann A, Reinhart K, Bredle DL, Specht M, Spies CD, Hannermann L. Epinephrine impairs splanchnic perfusion in septic shock. Crit Care Med. 1997;25:399-404.

18. Martikainen TJ, Tenhunen JJ, Giovannini I, Uusaro A, Roukonen E. Epinephrine induces tissue perfusion deficit in porcine endotoxin shock: evaluation by regional $\mathrm{CO}(2)$ content gradients and lactate-to-pyruvate ratios. Am J Physiol Gastrointest Liver Physiol. 2005;288:G586-92.

19. Deltere P, Valla DC. Ischemic cholangiopathy. J Hepatol. 2006:44:806-17.

20. Sonomura T. Optimal size of embolic material in transcatheter arteria embolization of the liver. Nihon Igaku Hoshasen Gakkai Zasshi. 1994; 54:489-99.

21. Doppman JL, Dunnick NR, Girton M, Fauci AS, Popovsky MA. Bile duct cysts secondary to liver infarcts: report of a case and experimental production by small vessel hepatic artery occlusion. Radiology. 1979;130:1-5

22. Differding JA, Underwood SJ, Van PY, Khaki RA, Spoerke NJ, Schreiber MA. Trauma induces a hypercoagulable state that is resistant to hypothermia as measured by thrombelastogram. Am J Surg. 2011;201:587-91.

23. Brohi K, Cohen MJ, Ganter MT, Schultz MJ, Levi M, Mackersie RC, et al. Acute coagulopathy of trauma: hypoperfusion induces systemic anticoagulation and hyperfibrinolysis. J Trauma. 2008;64:1211-7.

24. Floccard B, Rugeri L, Faure A, Saint Denis M, Boyle EM, Peguet O, et al. Early coagulopathy in trauma patients: an on-scene and hospital admission study. Injury. 2012:43:26-32.

25. Hunt BJ, Parrat RN, Segal HC, Sheikh S, Kallis P, Yacoub M. Activation of coagulation and fibrinolysis during cardiothoracic operations. Ann Thorac Surg. 1998;65:712-8.

26. Stahl RL, Duncan A, Hooks MA, Henderson JM, Millikan WJ, Warren WD. A hypercoagulable state follows orthotopic liver transplantation. Hepatology. 1990;12:553-8

27. Lison S, Weiss G, Spannagl M, Heindl B. Postoperative changes in procoagulant factors after major surgery. Blood Coagul Fibrinolysis. 2011;22:190-6.

28. Kirby DF, Blei AT, Rosen ST, Vogelzang RL, Neiman HL. Primary sclerosing cholangitis in the presence of a lupus anticoagulant. Am J Med. 1986;81:1077-80.
29. Viola S, Meyer M, Fabre M, Tounian P, Goddon R, Dechelotte P, et al. Ischemic necrosis of bile ducts complicating Schönlein-Henoch purpura. Gastroenterology. 1999;117:11-4

30. Le Thi HD, Valla D, Franco D, Wechsler B, De Gramont A, Auperin A, et al. Cholangitis associated with paroxysmal nocturnal hemoglobinuria: another instance of ischemic cholangiopathy? Gastroenterology. 1995; 109:1338-43.

31. Lamy $P$, Valla D, Bourgeois $P$, Rueff B, Benhamou JP. Primary sclerosing cholangitis and systemic lupus erythematosus. Gastroenterol Clin Biol. 1988;12:962-4.

32. Audan A, Bruley Des Varannes S, Georgelin T, Sagan C, Cloarec D, Serraz H, et al. Primary sclerosing cholangitis and systemic lupus erythematosus. Gastroenterol Clin Biol. 1995;19:123-6.

33. Chernysheva GA, Plotnikov MB, Smol'yakova VI, Avdoshin AD, Saratikov AS, Sutormina TG. Relationship between rheological and hemodynamic changes in rats with crush syndrome. Bull Exp Biol Med. 2000;130:1048-50.

34. Heidenhain C, Pratschke J, Puhl G, Neumann U, Pascher A, Veltzke-Schlieker W, et al. Incidence of and risk factors for ischemic-type biliary lesions following orthotopic liver transplantation. Transpl Int. 2010;23:14-22.

35. Moench C, Moench K, Lohse AW, Thies J, Otto G. Prevention of ischemic-type biliary lesions by arterial back-table pressure perfusion. Liver Transpl. 2003;9:285-9.

36. de Morais BS, Sanches MD, Ribeiro DD, Lima AS, de Abreu Ferrari TC Duarte MM, et al. Association between the use of blood components and the five-year mortality after liver transplant. Rev Bras Anestesiol. 2011;61:286-92.

37. Ramos E, Dalmau A, Sabaté A, Lama C, Lladó I, Figueras J, et al. Intraoperative red blood cell transfusion in liver transplantation: influence on patient outcome, prediction of requirements, and measures to reduce them. Liver Transpl. 2003;9:1320-7.

38. Van Bommel J, de Korte D, Lind A, Seigemund M, Trouwborst A, Verhoeven AJ, et al. The effect of the transfusion of stored RBCs on intestinal microvascular oxygenation in the rat. Transfusion. 2001; 41:515-23.

39. Fujita Y. Effects of PEEP on splanchnic hemodynamics and blood volume. Acta Anaesthesiol Scand. 1993;37:427-31.

40. Kubitz R, Wettstein M, Warskulat U, Häussinger D. Regulation of the multidrug resistance protein 2 in the rat liver by lipopolysaccharide and dexamethasone. Gastroenterology. 1999;16:401-10.

41. Trauner M, Arrese M, Soroka CJ, Ananthanarayanan M, Koeppel TA, Schlosser SF, et al. The rat canalicular conjugate export pump (Mrp2) is down-regulated in intrahepatic and obstructive cholestasis. Gastroenterology. 1997;113:255-64.

42. Vos TA, Hooiveld GJ, Koning $\mathrm{H}$, Childs $\mathrm{S}$, Meijer DK, Moshage $\mathrm{H}$, et al. Up-regulation of the multidrug resistance genes, Mrp1 and Mdr1b, and down-regulation of the organic anion transporter, Mrp2, and the bile salt transporter, Spgp, in endotoxemic rat liver. Hepatology. 1998;28:1637-44.

43. Hartmann G, Kim H, Piquette-Miller M. Regulation of the hepatic multidrug resistance gene expression by endotoxin and inflammatory cytokines in mice. Int Immunopharmacol. 2001;1:189-99.

44. Spirlì C, Nathanson MH, Fiorotto R, Duner E, Denson LA, Sanz JM, et al. Proinflammatory cytokines inhibit secretion in rat bile duct epithelium. Gastroenterology. 2001;121:156-69.

45. Rangel-Frausto MS, Pittet D, Costigan M, Hwang T, Davis CS, Wenzel RP. The natural history of the systemic inflammatory response syndrome (SIRS). A prospective study. JAMA. 1995;273:117-23.

46. Deltenre P, Valla DC. Ischemic cholangiopathy. J Hepatol. 2006; 44:806-17.

47. Sakrak O, Akpinar M, Bedirli A, Akyurek N, Aritas Y. Short and long-term effects of bacterial translocation due to obstructive jaundice on liver damage. Hepatogastroenterology. 2003;50:1542-6.

48. Sherlock S. Pathogenesis of sclerosing cholangitis: the role of nonimmune factors. Semin Liver Dis. 1991;11:5-10.

49. Leonhardt S, Veltz-Schlieker W, Adler A, Schott E, Eurich D, Faber W, et al. Secondary sclerosing cholangitis in critically ill patients: clinical presentation, cholangiography features, natural history, and outcome. Medecine. 2015;94:e2188.

50. Weber C, Kuhlencordt R, Grotelueschen R, Wedegaertner U, Ang TL, Adam $G$, et al. Magnetic resonance cholangiopancreatography in the diagnosis of primary sclerosing cholangitis. Endoscopy. 2008;40:739-45.

51. Voigtländer T, Leuchs E, Vonberg RP, Solbach P, Manns MP, Suerbaum S, et al. Microbiological analysis of bile and its impact in critically ill patients with secondary sclerosing cholangitis. J Infect. 2015;70:483-90.

52. Lazaridis KN, Strazzabosco M, Larusso NF. The cholangiopathies: disorders of biliary epithelia. Gastroenterology. 2004;127:1565-77.

53. Weisner RH, Porayko MK, Dickson ER, et al. Selection and timing of liver transplantation in primary biliary cirrhosis and primary sclerosing cholangitis. Hepatology. 1992;16:1290-9.

54. Farger $\mathrm{O}$, Malassagne $\mathrm{B}$, Sebagh $\mathrm{M}$, Bismuth $\mathrm{H}$. Primary sclerosing cholangitis: liver transplantation or biliary surgery. Surgery. 1995;117:146-55. 